\title{
Baleyssagues, Duras et Esclottes
}

Nicolas Rampnoux

URL : http://journals.openedition.org/adlfi/7569

ISSN : 2114-0502

Éditeur

Ministère de la culture

Référence électronique

Nicolas Rampnoux, "Baleyssagues, Duras et Esclottes », ADLFI. Archéologie de la France - Informations [En ligne], Aquitaine, mis en ligne le 01 mars 2007, consulté le 19 avril 2019. URL : http://

journals.openedition.org/adlfi/7569

Ce document a été généré automatiquement le 19 avril 2019

(c) Ministère de la Culture et de la Communication, CNRS 


\title{
Baleyssagues, Duras et Esclottes
}

\author{
Nicolas Rampnoux
}

Identifiant de l'opération archéologique : 025006

Date de l'opération : 2006 (PT)

1 En 2006, les trois communes du Lot-et-Garonne : Baleyssagues, Duras et Esclottes ont vu débuter un programme de prospection pédestre (en vue d'un mémoire de Master II à l'université Bordeaux III).Celui-ci a pour but d'estimer le potentiel archéologique de cette zone depuis longtemps délaissée par les chercheurs. Les sites des époques protohistoriques et gallo-romaines sont principalement ciblés.

2 Dans cette étude, le choix géographique est fondé sur deux critères : la proximité avec une voie navigable, le Dropt et le point de jonction de trois territoires: celui des Pétrucores, des Nitiobroges et des Vasates.

3 La première partie de la prospection a consisté en une vérification des sites déjà recensés. Nous sommes partis d'un total de vingt-trois sites déjà connus. Il s'avère que bon nombre ne sont que des indices documentaires et n'ont jamais donné lieu à une quelconque recherche approfondie. Sur le terrain ces informations, bien que souvent précises, ne permettent pas de confirmer par une simple étude de surface la présence de site. La difficulté de localisation des sites s'explique par deux raisons : l'ancienneté des données d'une part, souvent vieilles de plus de vingt ans et un terrain peu propice à une prospection pédestre.

4 À titre d'exemple, on peut citer les terres viticoles impossible à prospecter en l'état et couvrant des sites déjà référencés. Le site de « Ménéguerre - Grand Pasti » ou celui de «Toulouse - Maubourget », tous les deux dans la commune d'Esclottes sont couverts par des rangs de vignes.

5 En ce qui concerne les nouveaux sites découverts, on a recensé dix-huit indices de sites difficiles à valider, les informations recueillies étant insuffisantes pour réellement confirmer ou infirmer leur présence. Le lieu-dit « Mirathe » à Baleyssagues a livré comme indices trois silex d'origine indéterminée mais cependant le façonnage est 
indiscutablement anthropique. Nous avons également retrouvé des briques et des tuiles indatables dans l'état excepté un fragment de tegula.Cet élément étant unique, il ne peut en aucun cas constituer une preuve de la présence gallo-romaine.

6 À ces sites s'ajoutent dix autres que nous pouvons considérer comme tel. Leur datation a été estimée suivant les indices récoltés. Dans le cas du lieu-dit "Grand-Puy», des éléments gallo-romains ont été retrouvés ainsi que des restes de tuiles surcuites, qui présentent des traces de coulées, (éventuellement un creuset?).

7 À Duras, au lieu-dit «le point du Jour » des indices de structures ont été repérés. Un nombre important de moellons ont été regroupés au même endroit. Cependant, la plantation de pins qui occupe la parcelle rend impossible toute autre investigation de surface. À Duras également, des creusements dans le substrat calcaire sont visibles au lieu-dit «Fonbouet", ainsi qu'une structure en pierre sèche écroulée, à la fonction indéterminée. Cela peut être une carrière ou des habitats semi-troglodytiques ou encore les deux simultanément. D'autres excavations du substrat sont observables à Esclottes au lieu-dit «Champ du Roc» ou encore au «tennement de Terra ». On peut supposer qu'il s'en trouve d'autres dans les communes avoisinantes localisées au bord des plateaux.

D'un point de vue chronologique, une part importante des tessons semblent appartenir aux Temps Modernes, ou être légèrement antérieurs. Une assez grande quantité de tessons a été attribuée à l'époque gallo-romaine; en revanche, beaucoup d'objets ramassés ne sont pas datables (trop corrodés, trop petits ou non caractéristiques).

\section{INDEX}

operation Prospection thématique (PRT)

Index chronologique : Empire romain, Protohistoire, Temps Modernes

peuple Gaulois

Index géographique : Aquitaine, Lot-et-Garonne (47), Baleyssagues

Thèmes : brique, céramique gallo-romaine, céramique moderne, documentation, exploitation des données, localisation, moellon, navigation fluviale, rivière, silex, sylviculture, territoire, tuile, vigne

\section{AUTEURS}

NICOLAS RAMPNOUX

SUP 